\title{
Role of ultrasound guided fine needle aspiration biopsy in the diagnosis of hepatocellular carcinoma
}

\author{
G Sbolli, F Fornari, G Civardi, M Di Stasi, L Cavanna, E Buscarini, L Buscarini
}

\begin{abstract}
In 170 cases of hepatocellular carcinoma, ultrasound showed a high sensitivity in identifying focal liver lesions. Fine needle aspiration biopsy guided by ultrasound yielded a pathological diagnosis in the majority of cases. The advantages of this technique, its high diagnostic yield and low cost, render the older technique of blind percutaneous biopsy using a coarse needle obsolete. Laparoscopy retains its essential role in selected cases. Complementary use of fine needle aspiration biopsy under ultrasound guidance and laparoscopy assures the highest rate of diagnostic accuracy in hepatocellular carcinoma. We confirm the poor sensitivity of $\alpha$ fetoprotein
\end{abstract}

The incidence of hepatocellular carcinoma in some areas of Asia and Africa reaches 150 cases/ 100000 inhabitants per year; in these areas hepatocellular carcinoma is the most common neoplasm. In Europe, the United States, and Australia the reported incidence is less than 5 cases $/ 100000 .^{\prime}$ It is probably underestimated, however.

Hepatocellular carcinoma is more frequent in men and in the middle aged and elderly. But in the areas where hepatitis $B$ is endemic, the prevalence of this tumour is higher and the average patient age lower. Known risk factors are hepatitis B virus, alcohol, and liver cirrhosis. ${ }^{2}$

$\alpha$ Fetoprotein concentrations show poor sensitivity for early diagnosis of hepatocellular carcinoma. Detection is facilitated by ultrasonography, whereas pathological diagnosis is possible with guided fine needle biopsy. ${ }^{34}$ The diagnostic accuracy of cytology in hepatocellular carcinoma has now been confirmed by several studies. ${ }^{5-8}$

Early diagnosis is important in order to modify the extremely poor prognosis of this tumour by surgical intervention. In this paper we report data from 170 consecutive patients with hepatocellular carcinoma seen in our department. The aim is to point out the diagnostic role of ultrasound guided fine needle aspiration biopsy in the diagnosis of hepatocellular carcinoma.

$1^{2}$ Divisione di Medicina, Servizio di Gastroenterologia, Ospedale di Piacenza, via Taverna 49, 29100Piacenza, Italy G Sbolli F Fornari G Civardi M Di Stasi L Cavanna E Cavanna L Buscarini Correspondence to: G Sbolli. 8 January 1990
Methods
We report on 170 consecutive patients with
hepatocellular carcinoma seen from June 1981 to
February 1989 . Hepatitis B virus markers (Elisa-
Ria Abbott Laboratories) and $\alpha$ fetoprotein
(Enzymost Immunoassay Behring Institute)
assays were performed, as well as routine liver
function tests. In all patients abdominal ultra-
sonography was performed with real-time equip-
ment (Hitachi EUB 22,26 and Aloka SSD 650

Methods
We report on 170 consecutive patients with
hepatocellular carcinoma seen from June 1981 to
February 1989 . Hepatitis B virus markers (Elisa-
Ria Abbott Laboratories) and $\alpha$ fetoprotein
(Enzymost Immunoassay Behring Institute)
assays were performed, as well as routine liver
function tests. In all patients abdominal ultra-
sonography was performed with real-time equip-
ment (Hitachi EUB 22,26 and Aloka SSD 650

Methods
We report on 170 consecutive patients with
hepatocellular carcinoma seen from June 1981 to
February 1989 . Hepatitis B virus markers (Elisa-
Ria Abbott Laboratories) and $\alpha$ fetoprotein
(Enzymost Immunoassay Behring Institute)
assays were performed, as well as routine liver
function tests. In all patients abdominal ultra-
sonography was performed with real-time equip-
ment (Hitachi EUB 22,26 and Aloka SSD 650

Methods
We report on 170 consecutive patients with
hepatocellular carcinoma seen from June 1981 to
February 1989 . Hepatitis B virus markers (Elisa-
Ria Abbott Laboratories) and $\alpha$ fetoprotein
(Enzymost Immunoassay Behring Institute)
assays were performed, as well as routine liver
function tests. In all patients abdominal ultra-
sonography was performed with real-time equip-
ment (Hitachi EUB 22,26 and Aloka SSD 650

Methods
We report on 170 consecutive patients with
hepatocellular carcinoma seen from June 1981 to
February 1989 . Hepatitis B virus markers (Elisa-
Ria Abbott Laboratories) and $\alpha$ fetoprotein
(Enzymost Immunoassay Behring Institute)
assays were performed, as well as routine liver
function tests. In all patients abdominal ultra-
sonography was performed with real-time equip-
ment (Hitachi EUB 22,26 and Aloka SSD 650

Methods
We report on 170 consecutive patients with
hepatocellular carcinoma seen from June 1981 to
February 1989 . Hepatitis B virus markers (Elisa-
Ria Abbott Laboratories) and $\alpha$ fetoprotein
(Enzymost Immunoassay Behring Institute)
assays were performed, as well as routine liver
function tests. In all patients abdominal ultra-
sonography was performed with real-time equip-
ment (Hitachi EUB 22,26 and Aloka SSD 650

Methods
We report on 170 consecutive patients with
hepatocellular carcinoma seen from June 1981 to
February 1989 . Hepatitis B virus markers (Elisa-
Ria Abbott Laboratories) and $\alpha$ fetoprotein
(Enzymost Immunoassay Behring Institute)
assays were performed, as well as routine liver
function tests. In all patients abdominal ultra-
sonography was performed with real-time equip-
ment (Hitachi EUB 22,26 and Aloka SSD 650

Methods
We report on 170 consecutive patients with
hepatocellular carcinoma seen from June 1981 to
February 1989 . Hepatitis B virus markers (Elisa-
Ria Abbott Laboratories) and $\alpha$ fetoprotein
(Enzymost Immunoassay Behring Institute)
assays were performed, as well as routine liver
function tests. In all patients abdominal ultra-
sonography was performed with real-time equip-
ment (Hitachi EUB 22,26 and Aloka SSD 650 with $3-3 \cdot 5 \mathrm{MHz}$ linear or convex probes). In some cases computed tomography, selective liver angiography, and laparoscopy were carried out. Ultrasound guided fine needle aspiration biopsy (Chiba needle, 22 gauge) was performed according to the technique we previously described. ${ }^{9}$ Aspiration was performed with an aspir gun with short forward and backward movements visualising the bright echo of the needle tip in the target.

All patients had platelet counts of over $70 \times 10^{9} / 1$ and prothrombin times (\% of the control) higher than $50 \%$ (international normalised ratio $1 \cdot 30$ ).

A cytology sample, stained by a rapid MayGrünwald-Giemsa method (Merck Hemacolor), was examined immediately and a subsequent biopsy was performed only when the first specimen was inadequate. ${ }^{10}$ The other samples, stained with Papanicolaou and May-GrünwaldGiemsa, were examined by an experienced cytologist for definitive diagnosis.

In three cases of well differentiated hepatocellular carcinoma the cytological suspicion was confirmed by a cutting fine needle biopsy (Surecut). Verification of the cytological diagnosis was obtained by histological examination of samples obtained during laparoscopy (11 cases), surgery (eight cases), angiography (15 cases); by $\alpha$ fetoprotein concentrations $(>500 \mathrm{ng} / \mathrm{ml})(35$ cases); and by correlation with clinical, laboratory, and imaging data (63 cases).

The cytological diagnoses were categorised as true positives, true negatives, false positives, false negatives, and non-diagnostic (inadequate specimens/insufficient material). In this way we have evaluated the sensitivity, specificity, accuracy, and positive and negative predictive value of fine needle aspiration biopsy in hepatocellular carcinoma and calculated the significance of the results using the $\chi^{2}$ test.

\section{Results}

The study group comprised 131 men and 39 women (male to female ratio $3: 1$ ). The mean age was 65.4 years $(64.5$ years for men and 68.8 years for women), range 40-83 years.

Liver cirrhosis was ascertained in 149 of 170 $(87 \cdot 6 \%)$. In 66 cases diagnosis was made by histology or laparoscopy, or both, and in the other cases by clinical, laboratory, and ultrasound data. Serum hepatitis B virus markers were measured in 160 of 170 cases and 22 patients were $\mathrm{HBsAg}$ positive (13.7\%). $\alpha$ Fetoprotein was measured in 165 of 170 patients: normal concentrations were found in 63 cases $(38 \cdot 2 \%), 21-500 \mathrm{ng} / \mathrm{ml}$ in 54 cases $(32 \cdot 8 \%)$, and $>500 \mathrm{ng} / \mathrm{ml}$ in 48 cases (29\%). $\alpha$ Fetoprotein concentrations did not vary significantly with 
tumour size, cytology, or hepatitis B virus markers.

Ultrasonography was carried out in all patients: the liver lesion was detected in 167 of $170(98 \%)$; the nature of the lesion was misinterpreted in three cases. The tumour appeared as a single nodule in 77 cases (the diameter was less than $5 \mathrm{~cm}$ in 34 cases $(20.3 \%)$ and less than $3 \mathrm{~cm}$ in 16 cases $(9 \cdot 5 \%)$ ), as multiple nodules in 51 cases, and was diffuse in 39 cases; $46.6 \%$ of the lesions with a diameter of less than $5 \mathrm{~cm}$ appeared hypoechoic.

Computed tomography, performed in 40 patients, detected lesions in $86 \%$ of the cases; angiography, carried out in 28 patients, showed $100 \%$ accuracy.

Altogether, 138 patients underwent ultrasound guided fine needle aspiration biopsy. The diagnosis of hepatocellular carcinoma was obtained in 132 cases (sensitivity 95.6\%; specificity $100 \%) ; 68$ cases showed a well differentiated cell tumour, in 50 the tumour was poorly differentiated, and 14 showed a large pleiomorphic cell type. We report only one case of bleeding after biopsy, which required blood transfusion $(500 \mathrm{ml})$.

In the other 38 patients (six fine needle biopsy false negatives and 32 remaining patients without fine needle biopsy) a definitive diagnosis was reached with ultrasound guided $2 \cdot 1 \mathrm{~mm}$ tru-cut biopsy (one case); with laparoscopy and associated biopsy ( 13 cases); on the basis of clinical and laboratory data and ultrasonography (20 cases); and at necropsy (four cases).

In this series the 44 laparoscopies were performed to establish the initial diagnosis, to correct false negative fine needle biopsy, to determine presurgical staging, and to check the true positives of fine needle biopsy during its introductory phase into our department. The sensitivity of laparoscopy was $70 \cdot 5 \%$. The sensitivity was better when this technique was used to check the true positives of fine needle biopsy or to correct false negatives where laparoscopy was not performed in the cases with deeply located lesions.

\section{Discussion}

Laboratory diagnosis of hepatocellular carcinoma is principally based on $\alpha$ fetoprotein titres, even though many workers point out the poor sensitivity of this marker for early diagnosis. ${ }^{11-13}$ We found normal values in $38.2 \%$ of the cases and highly diagnostic values in a quarter of the patients. We did not find a significant correlation between $\alpha$ fetoprotein values and hepatocellular carcinoma cytology or tumour dimensions.

In the present series the percentage of the single hepatocellular carcinoma lesions with a diameter of less than $3 \mathrm{~cm}$ is small, $9 \cdot 5 \%$. In another Italian study ${ }^{13}$ the percentage was $10 \cdot 5 \%$.

To detect hepatocellular carcinoma we always performed ultrasonography, whose sensitivity is considered to be very good. ${ }^{12}{ }^{14}$ For lesions of less than $2 \mathrm{~cm}$ it gave a diagnostic accuracy higher than computed tomography and similar to angiography. ${ }^{15}$ Recent reports (on a small series of hepatocellular carcinoma) attribute a higher diagnostic accuracy to computed tomography and magnetic resonance imaging than to ultrasonography. Ultrasonography must nevertheless be considered the primary technique for safety, economy, and good definition of the vascular and biliary trees. ${ }^{1617}$ In addition, it can optimally guide the biopsy needle.

In selected cases ultrasonography with pulsed Doppler is useful to avoid haemorrhagic complications of fine needle aspiration biopsy guided by ultrasonography either by showing the vascular nature of the target lesion, or by showing a highly vascular area around the mass. ${ }^{18}$

Pathological diagnosis was reached in most of our patients with ultrasound guided fine needle biopsy, which showed a high diagnostic accuracy with a sensitivity of $95.6 \%$ in 138 patients. Bret $e t$ $a l^{19}$ used the procedure in 159 cases of hepatocellular carcinoma, obtaining a diagnostic sensitivity of $84 \%$ ( $92 \%$ for sensitivity in predicting the malignancy).

The cytological diagnosis of hepatocellular carcinoma can be difficult in well differentiated cell types. In fact, four of our six false negative cases were well differentiated cell type hepatocellular carcinoma (the other two cases were technically difficult to approach: small and deep target lesions). Recent reports cite a diagnostic accuracy of cytology (sampling under laparoscopic guide) ranging from $93 \%{ }^{7}$ to $100 \% .^{8}$ Bret et $a l^{19}$ point out the usefulness of comparing hepatocytes sampled from tumour and from normal areas of the liver. Pedio et $a l^{20}$ have shown the importance of naked nuclei, which show the same alterations as intact tumour hepatocytes. The phenomenon seems to be characteristic of hepatocellular carcinoma and is absent in liver cirrhosis.

To improve diagnostic accuracy Limberg et $a l^{21}$ suggest the use of cutting fine needle biopsy. Their results, however, are similar to ours and to those of Bret et al. ${ }^{19}$ We have used cutting fine needle biopsy in only three cases of well differentiated hepatocellular carcinoma to correct an equivocal cytological 'negative.' Such a manoeuvre, however, increases the cost of the investigation and may increase the risk to the patient. In our series we had one case of bleeding. In the series of Bret $e t a l^{19}$ there were four cases of bleeding, one of which was lethal.

In a recent series ${ }^{22}$ reporting the complications of abdominal fine needle biopsy, the mortality was $0.018 \%$ (the two deaths were due to haemoperitoneum in cases of hepatocellular carcinoma with cirrhosis). We agree with Hall-Craggs and Lees $^{23}$ that the use of the cutting fine needle has to be reserved for those cases not diagnosed by cytology. We emphasise that the rapid staining of the material aspirated during ultrasound guided biopsy can reduce the number of biopsy specimens required from each patient without impairing the diagnostic accuracy ${ }^{10}$ and can thereby reduce the complication rate associated with the biopsy.

We point out that ultrasound guided fine needle biopsy should replace blind percutaneous biopsy $^{24}$ for typing of hepatocellular carcinoma and other focal liver lesions. The introduction of fine needle biopsy has modified the indications for laparoscopy. ${ }^{25}$ 
For the initial diagnosis we used laparoscopy in selected cases: suspected hepatocellular carcinoma with negative or inconclusive ultrasound, lesions with ultrasound features resembling subcapsular haemangioma, or cases where there were difficulties in performing fine needle biopsy. Laparoscopy is advisable to correct false negatives and for presurgical staging. Moreover, it is useful for the study of equivocal lesions in a cirrhotic liver with adenomatous hyperplasia. ${ }^{26}$

We think that ultrasonography is a good method for identifying hepatocellular carcinoma, while ultrasound guided fine needle aspiration biopsy seems to be the first choice of the invasive technique. The indications for laparoscopy are thereby reduced, but it retains an essential role in selected cases. The integration of these two techniques gives excellent diagnostic accuracy. ${ }^{27}$

We thank Dr Nicola Strickland, Hammersmith Hospital, London, for her kind cooperation in revising the English text.

1 Rustigi VK. Epidemiology of hepatocellular carcinoma. Hepatocellular carcinoma. Ann Intern Med 1988; 108: 390 401 .

2 Di Bisceglie AM. Etiology of hepatocellular carcinoma. Ann Intern Med 1988; 108: 390-401.

3 Tao L, Ho C, McLoughlin M, et al. Cytologic diagnosis of hepatocellular carcinoma by fine needle aspiration biopsy. Cancer 1984; 53: 547-52.

4 Buscarini L, Sbolli G, Cavanna L, et al. Clinical and diagnostic features of 67 cases of hepatocellular carcinoma. Oncology 1987; 44: 93-7

5 Tao LC, Donat EE, Ho CS, McLoughlin MJ. Percutaneous fine needle aspiration biopsy of the liver. Cytodiagnosis of hepatic cancer. Acta Cytol 1979; 23: 287-91.

6 Suen KC. Diagnosis of primary hepatic neoplasm by fineneedle aspiration cytology. Diagn Cytopathol 1986; 2: 99109.

7 Cussó X, Marti-Vicente A, Monés-Xiol J, Vilardel F. Laparoscopic cytology. An evaluation. Endoscopy 1988; 20: 102-3.

8 Jeffers L, Spielgman G, Reddy R, et al. Laparoscopically directed fine needle aspiration for the diagnosis of hepatocellular carcinoma: a safe and accurate technique. Gastrointest Endosc 1988; 34: 235-7.

9 Fornari F, Cavanna L, Civardi G, et al. Ultrasonically guided fine-needle aspiration biopsy: first stage invasive procedure in the diagnosis of focal lesions of the liver. Ital $\mathcal{F}$ Gastro enterol 1985; 17: 246-51.

10 Civardi G, Fornari F, Cavanna L, Di Stasi M, Sbolli G Buscarini $L$. Value of rapid staining and assessment of ultrasound-guided fine needle aspiration biopsies. Acta Cyto 1988; 32: $552-4$.

11 Chen D, Sung J, Sheu J, et al. Serum alpha-fetoprotein in the early stage of human hepatocellular carcinoma. Gastroenterolog $1984 ; 86$ : 1404-9.

12 Sheu JC, Sung JL, Chen DS, et al. Early detection of hepatocellular carcinoma by real-time ultrasonography. A prospective study. Cancer 1985; 56: 660-6.

13 Gandolfi L, Solmi L, Bertoni F, et al. Small hepatocellular carcinoma: an Italian experience. Hepatogastroenterology 1987; 34: 100-2.

14 Okuda K. Early recognition of hepatocellular carcinoma Hepatology 1986; 6: 729-38.

15 Kudo M, Hirasa M, Takakuwa $\mathbf{H}$, et al. Small hepatocellular carcinomas in chronic liver disease: detection with SPECT Radiology 1986; 159: 697-703.

16 Curati WL, Halevy A, Gibson RN, et al. Ultrasound, CT and MRI comparison in primary and secondary tumors of the liver. Gastrointest Radiol 1988; 13: 123-8.

17 Devred PH, Ghersi A, Roumieu G, et al. Intérêt de l'IRM dans l'exploration des carcinomes hépatocellulaires. Etude prél'exploration des carcinomes hépatocellulaires. Etude

18 McGahan JP, Anderson NW. Pulsed doppler sonography as an aid in ultrasound-guided aspiration biopsy. Gastrointes an aid in ultrasound-guide

19 Bret PM, Labadie M, Bretagnolle M, et al. Hepatocellular carcinoma: diagnosis by percutaneous fine-needle biopsy. Gastrointest Radiol 1988; 13: 253-5.

20 Pedio G, Landolt U, Zöbeli L, Gut D. Fine needle aspiration of the liver. Significance of hepatocytic naked nuclei in the diagnosis of hepatocellular carcinoma. Acta Cytol 1988; 32: $437-42$.

21 Limberg B, Höpker WW, Kommerel B. Histologic differential diagnosis of focal liver lesions by ultrasonically guided fine needle biopsy. Gut 1987; $28: 237-41$.

22 Fornari $\mathrm{F}$ Civardi G Cavanna 2 et al. Complications of ultrasonically guided fine needle abdominal biopsy: results ultrasonically guided fine needle abdominal biopsy: results of a multicentre Italian study and

23 Hall-Craggs MA, Lees WR. Fine needle biopsy: cytology, histology or both? Gut 1987; 28: 233-6.

24 Villa E, Baldini MG, Pasquinelli C, et al. Risk factors for hepatocellular carcinoma in Italy. Male sex, hepatitis B, virus non-A non-B infection and alcohol. Cancer 1988; 62: 611-5.

25 Buscarini L, Sbolli G, Civardi G, et al. Does ultrasonically guided biopsy modify indications to laparoscopy in hepatology? Acta Endosc 1987; 17: 85-8.

26 Buscarini L, Fornari F, Cavanna L, et al. Diagnostic problem of hyperplastic regenerative nodule in cirrhotic liver. Acta Endosc 1987; 17: 233-9.

27 Fornari F, Rapaccini GL, Cavanna L, et al. Diagnosis of hepatic lesions: ultrasonically guided fine needle biopsy or laparoscopy? Gastrointest Endosc 1988; 34: 231-4.

28 Miller DA, Carrasco CH, Katz RL, Cramer FM, Wallace S, Charnsangavej $C$. Fine needle aspiration biopsy: the role of immediate cytologic assessment. AfR 1986; 147: 155-8. 